\title{
PAPER
}

\section{Clinical application of neuroimaging in epilepsy}

\section{U C Wieshmann}

Correspondence to: Dr U C Wieshmann, The Walton Centre for

Neurology and

Neurosurgery, Lower Lane, Liverpool L9 7L, UK; wieshmann@vizzavi.net

Received

7 October 2002

Accepted

24 November 2002

\begin{abstract}
Objective: To evaluate the use of neuroimaging in clinical practice and to assess the prevalence of detected structural abnormalities in epilepsy patients in a clinical set up.

Methods: 919 outpatients were identified and the scan results reviewed. A total of 677 patients had chronic active epilepsy (88 had idiopathic generalised epilepsy (IGE), 588 had localisation related epilepsy, one had symptomatic generalised epilepsy), 57 had a single epileptic seizure, 46 were in remission, and 139 had non-epileptic attacks.

Results: 391 patients had no scan 153 patients in this group had IGE, 182 had localisation related epilepsy, one had generalised symptomatic epilepsy, 18 had single epileptic attacks, 21 were in remission, 116 had non-epileptic attacks). Altogether 528 patients had a scan, the results were not available in 33, 163 had $x$ ray computed tomography (CT) only, 178 had standard magnetic resonance imaging (MRI) (slice thickness $5 \mathrm{~mm}$ ), and 154 had high resolution MRI (including a T1 weighted sequence with $1.5 \mathrm{~mm}$ thick slices). Some 252 of 495 scans (51\%) were abnormal. Abnormalities were hippocampal sclerosis $(n=128)$, atrophy or non-specific white matter lesions $(n=35)$, vascular abnormalities $(n=27)$, tumours $(n=25)$, brain damage $(n=24)$, malformations of cortical development $(n=13)$. Excluding atrophy and non-specific white matter lesions the prevalence of detected abnormalities was $54 \%$ in localisation related epilepsy, $18 \%$ in single seizure patients, $16 \%$ in epilepsy in remission, and $0 \%$ in IGE and non-epileptic attacks.

Conclusions: Abnormalities were detected in more than half of all patients with localisation related epilepsy, and in about one in five patients with single seizures or epilepsy in remission. Many patients had no scan or only CT or standard MRI. The true prevalence of structural abnormalities may be have been higher. Scanning did not add any information in patients with IGE or non-epileptic attacks.
\end{abstract}

S ructural cerebral abnormalities can often be detected in epilepsy patients with modern neuroimaging techniques. Tumours, infarcts, and major malformations are usually detectable on $x$ ray computed tomography (CT) imaging. Magnetic resonance imaging (MRI) using appropriate sequences also permits the detection of more subtle abnormalities such as hippocampal sclerosis, ${ }^{1-7}$ subependymal nodular and band heterotopia, focal cortical dysplasia and small tumours including dysembryoplastic neuroepithelial tumours $^{8}$ (for review see Duncan ${ }^{9}$ ). Imaging studies performed at research centres suggested a high prevalence of structural abnormalities in patients with epilepsy. ${ }^{10}$ The results of research studies are not directly applicable to clinical routine because such studies differ from clinical routine in various ways. Research studies may suffer from selection bias, favouring patients with potential cerebral abnormalities. ${ }^{11}$ On the other hand, the imaging modalities applied in research studies are likely to be optimal for the detection of abnormalities and the scans are likely to be reviewed by specialists with an interest in epilepsy. This may not necessarily apply for clinical routine work. Neuroimaging guidelines have been published ${ }^{12}$ but the use of neuroimaging and the prevalence of detected abnormalities in a clinical environment are unknown. The aim of this paper was to review the use of neuroimaging and to assess the prevalence of detected structural abnormalities in a clinical hospital outpatient setting.

\section{METHODS}

For this cross sectional study we included patients with chronic active epilepsy, a single epileptic seizure, epilepsy in remission (no seizures for two years or longer) or nonepileptic seizures. For the purpose of this study patients suffering from non-epileptic attack disorder and patients with faints or other transient non-epileptic episodes were summarised under non-epileptic seizures. Chronic active epilepsies (recurrent seizures despite anticonvulsive treatment) were further classified as idiopathic generalised epilepsy (IGE) or other forms of epilepsy. ${ }^{13}$

The imaging reports were reviewed. It was clarified who produced the report (that is, a trained neuroradiologists or a general radiologist). The imaging modality was identifiedthat is, cranial $x$ ray computed tomography, standard MRI with slice thickness $5 \mathrm{~mm}$ (standard MRI), or high resolution MRI with one Tl weighted sequence with slice thickness 1.5 $\mathrm{mm}$.

The neuroradiological findings were classified as normal, consistent with hippocampal sclerosis, vascular abnormality (including infarct, arterio venous malformation, cavernous haemangioma), tumour, malformation of cortical development (including focal cortical dysplasia, schizencephaly, subependymal nodular and band heterotopia), brain damage, or non-specific abnormality (including white matter lesions).

\section{RESULTS}

\section{Clinical data}

A total of 919 patients were identified. The mean age was 39.7 years (median 39, minimum 15, maximum 87 years, standard deviation 14.2 years). Some 603 were seen at specialist epilepsy clinics (190 had been considered for epilepsy surgery), 316 patients were seen at general neurology clinics by one neurologist. Altogether 677 patients had chronic active epilepsy, 88 patients had IGE, 589 patients suffered from epilepsies other than IGE (all but one patient in this group had localisation related cryptogenic or symptomatic epilepsy, there were no patients with Rolandic epilepsy). Fifty seven patients

Abbreviations: IGE, idiopathic generalised epilepsy; CT, computed tomography; MRI, magnetic resonance imaging 


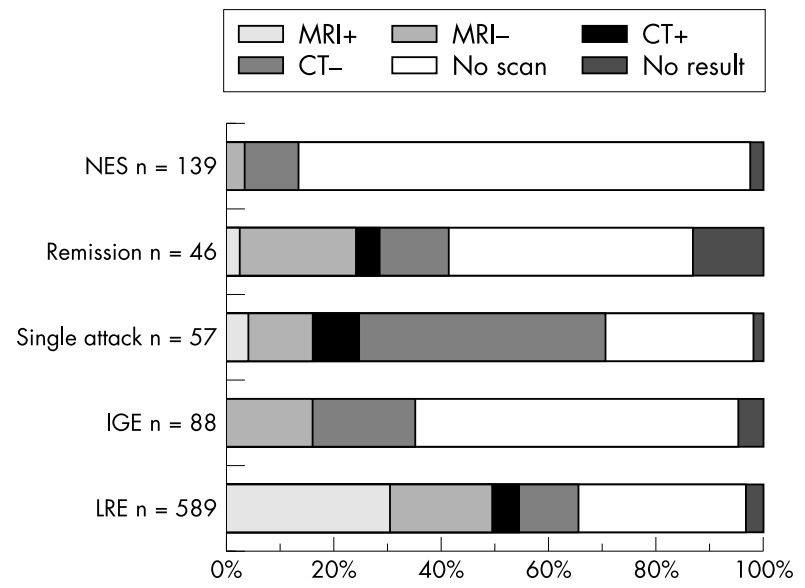

Figure 1 Stacked graph showing the number of patients with abnormal scans, normal scans, no scans, and unavailable results as a proportion of the total number for the different goups (localisation related epilepsy $(\mathrm{LRE})^{*}$, idiopathic generalised epilepsy (IGE), epilepsy in remission (Remission), single epileptic attack (Single attack), non-epileptic attacks (NES)). Only abnormalities relevant to epilepsy were included. (Hippocampal sclerosis, tumours, malformations, vascular abnormalities, non-specific white matter lesion, and atrophy were not included in this graph.) MRI+, abnormality on MRI detected; MRI-, no relevant abnormality detected; CT + , abnormality detected; CT-, no abnormality detected; no scan, no imaging performed; no result, scan result not available. ${ }^{*}$ LRE includes the single patient with symptomatic generalised epilepsy.

had single epileptic attacks, 46 were in remission, and 139 patients were classified as suffering from non-epileptic attacks. There was no overlap between the groups. The patients in remission were not included in the categories of generalised or focal epilepsy.

A total of 391 patients had no scan (53 patients had IGE, 182 had localisation related epilepsy, one had symptomatic generalised epilepsy, 18 had single epileptic attacks, 21 were in remission, 116 had non-epileptic attacks.) There were no obvious differences in practice between general neurology clinic and specialist epilepsy clinic.

\section{Neuroimaging data}

A total of 528 patients had a scan. The results of 33 scans were not available. In one patient the study was incomplete because of claustrophobia these patients were excluded from further analysis. The scan results of 495 patients were reviewed (mean age 40.1 years, standard deviation 13.5 years). Altogether 163 patients had CT only. A total of 178 patients had a standard MRI almost all patients in this group had also a CT scan. Some 154 patients had high resolution MRI (including a coronal Tl weighted sequence with slice thickness $1.5 \mathrm{~mm}$ ) in addition to CT and standard MRI. All 154 had been considered for epilepsy surgery (fig 1).
CT imaging was performed on a Philips CT scanner. Intravenous contrast was given if a focal onset was indicated on the request form. Axial images were obtained. Standard MRI was performed on a Siemens Magnetom or a GE 1.5 Tesla scanner. Sagittal T1 weighted images and axial T2 weighted and proton density weighted images were obtained in all patients. In some patients coronal $\mathrm{Tl}$ weighted images and coronal fluid attenuated inversion recovery (FLAIR) images were also obtained. All images had a slice thickness of $5 \mathrm{~mm}$ (typical matrix 192×256 and typical FOV $25 \mathrm{~cm}$. Intravenous contrast was given in a minority of patients (usually with mass lesions). A small group of patients had CT or standard MRI investigations using similar protocols at other hospitals. High resolution MRI (slice thickness $1.5 \mathrm{~mm}$ ) imaging was performed on a GE $1.5 \mathrm{~T}$ scanner. Coronal Tl weighted contiguous images with $1.5 \mathrm{~mm}$ slice thickness and coronal T2 weighted, proton density weighted, and FLAIR images with 5 $\mathrm{mm}$ slice thickness were obtained. Measurements of hippocampal $\mathrm{T} 2$ relaxation time and volume were performed using previously described methods. Normal limits of hippocampal volume and $\mathrm{T} 2$ relaxation time were established from 73 controls. Hippocampal measurements in a patient outside the $99 \%$ confidence intervals in controls were considered abnormal. All images had been reported by neuroradiologists.

\section{Scan results}

Some 252 of the 495 reviewed scan results (51\%) were abnormal.

\section{Chronic active localisation related epilepsy}

Abnormalities were most frequently detected in this group (58\% abnormalities). This group had the most favourable MRI:CT ratio of about 3 to 1 and had all but two high resolution MRI (slice thickness $1.5 \mathrm{~mm}$ ) scans.

\section{Single attack}

Abnormalities were detected in one of three of all patients with single epileptic attacks, most had non-specific abnormalities or cerebrovascular accidents. Two had tumours. One patient (of four patients under 20) was a 17 year old with a single generalised nocturnal seizure. This patient had a presumed oligodendroglioma in the right posterior temporal region. There were no signs on examination, the EEG was normal. The lesion was detected on CT and confirmed on standard MRI. Another 29 year old patient in the single attack group had a large right temporal arterio venous malformation. The initial investigation in this patient was a standard MRI, CT was not performed.

\section{Remission}

In more than $80 \%$ no abnormality was detected. Abnormalities were usually non-specific, two had vascular lesions (there was one patient who had an excision of an arterio venous malformation and one had a cerebrovascular accident) one patient had brain damage.

Table 1 Abnormal scans, excluding atrophy and non-specific white matter lesions

\begin{tabular}{lllll}
\hline & \multicolumn{3}{l}{ Abnormal/total (\%) } & \\
\cline { 2 - 5 } & All scans & CT only & sMRI & hrMRI \\
\hline LRE & $207 / 387(54)$ & $28 / 93(30)$ & $69 / 142(49)$ & $110 / 152(72)$ \\
IGE & $0 / 31(0)$ & $0 / 17(0)$ & $0 / 13(0)$ & $0 / 1(0)$ \\
Single & $7 / 40(18)$ & $5 / 31(16)$ & $2 / 9(22)$ & N/A \\
Remission & $3 / 19(16)$ & $2 / 8(25)$ & $1 / 11(9)$ & N/A \\
NES & $0 / 18(0)$ & $0 / 14(0)$ & $0 / 3(0)$ & $0 / 1(0)$ \\
Total & $217 / 495(44)$ & $35 / 163(22)$ & $72 / 178(40)$ & $110 / 154(71)$ \\
\hline
\end{tabular}

$\mathrm{CT}$, computed tomography; sMRI, standard magnetic resonance imaging with $5 \mathrm{~mm}$ thick slices; hrMRI, high resolution MRI with $1.5 \mathrm{~mm}$ thick slices; LRE, localisation related epilepsy; IGE, idiopathic generalised epilepsy; Remission, epilepsy in remission; Single, single epileptic attack; NES, non-epileptic attack. 
Table 2 Detected abnormalities

\begin{tabular}{lllllll}
\hline & HS & VAS & Tumour & DAM & MCD & Non-spec \\
\hline LRE & $128(33 \%)$ & $20(5 \%)$ & $23(6 \%)$ & $23(6 \%)$ & $13(3 \%)$ & $21(5 \%)$ \\
IGE & 0 & 0 & 0 & 0 & 0 & 1 \\
Single & 0 & 5 & 2 & 0 & 0 & 5 \\
Remission & 0 & 2 & 0 & 1 & 0 & 5 \\
NES & 0 & 0 & 0 & 0 & 0 & 3 \\
Total & 128 & 27 & 25 & 24 & 13 & 35 \\
\hline
\end{tabular}

HS, hippocampal sclerosis; VAS, vascular abnormalities, including arterio-venous malformation; DAM, brain damage; MCD, malformation of cortical development; Non-spec, non-specific white matter lesions and non-specific atrophy. Other abbreviations as for table 1 .

\begin{tabular}{llll} 
Table 3 & Abnormality and imaging modality \\
\hline & CT only & sMRI & hrMRI \\
\hline HS & 0 & 20 & 108 \\
VAS & 13 & 14 & 0 \\
Tumour & 4 & 19 & 2 \\
DAM & 18 & 6 & 0 \\
MCD & 0 & 13 & 0 \\
Non-spec & 17 & 18 & 0 \\
\hline
\end{tabular}

Abbreviations as for tables 1 and 2 .

\section{Non-epileptic attacks}

Only in $17 \%$ of patients with non-epileptic attacks were abnormalities detected, all were non-specific white matter lesions.

\section{IGE}

All but one IGE patient had normal scan reports. The detected abnormality was a single small non-specific white matter lesion in the brain stem of uncertain significance (table 1).

\section{Detected abnormalities}

The most commonly detected abnormality was hippocampal sclerosis $(n=128)$, followed by non-specific abnormalities $(n=35)$, vascular abnormalities $(n=27)$, tumours $(n=25$, of which were four meningiomas), brain damage $(n=24)$, malformations of cortical development $(n=13)$. Dual abnormality was described in two patients (table 2).

Hippocampal sclerosis was usually detected with high resolution MRI, other abnormalities were infrequent in this group of patients who were all considered for epilepsy surgery. There was one patient who had a small temporal glioma, which was only detected on high resolution MRI. Hippocampal sclerosis was occasionally detected with standard MRI, but not CT (table 3).

The standard MRI scans of two patients were initially reported as normal, but on review found to be abnormal. One patient had suffered from refractory cryptogenic localisation related epilepsy and learning disability and died a sudden unexplained death. A postmortem examination revealed hippocampal sclerosis, on review of the standard MRI with the knowledge of the result postmortem examination the left hippocampus was felt to be smaller and the white matter of the left hemisphere returned a high signal. Another patient suffered from refractory cryptogenic localisation related epilepsy. The patient had a mild learning disability but no family history, or cutaneous stigmata of tuberous sclerosis. His standard MRI was initially reported as normal but a subsequent CT showed two periventricular calcifications consistent with tubers. The CT prompted a review of the standard MRI, it was felt that there was an area of subtle malformation of cortical development.

In one patient standard MRI showed a dilatation of the right temporal horn, quantitative measurements of the hippocampus were within normal limits. The patient under- went a right temporal lobectomy, histological examination showed widespread gliosis involving white matter, the patient was not seizure free but experienced a $60 \%$ seizure reduction.

\section{DISCUSSION}

A striking finding of our study was that a high proportion of patients had no scan. In about 43\% (391 patients) no evidence of previous scanning was found. About $30 \%$ of all patients with localisation related epilepsy were not scanned. The rationale for imaging the brain of patients developing epilepsy is first to identify underlying abnormalities such as vascular lesions and tumours that require specific treatment and second to assist the formulation of syndromic and aetiological diagnosis. ${ }^{12}$ Our own data showed that in patients with localisation related epilepsy who were scanned, the prevalence of structural abnormalities was high. More than half of all scans were abnormal. A third of all patients had hippocampal sclerosis. Tumours, vascular lesions, and brain damage were present in about 5\% and malformations of cortical development were detected in 3\% of all patients with localisation related epilepsy. Similar overall abnormalities (61\%) and tumour prevalence $(6 \%)$ were found in a population based study. ${ }^{14}$ In the subgroup of our patients with localisation related epilepsy who were scanned with high resolution MRI, the prevalence of detected abnormalities was almost $80 \%$, similar to the prevalence reported by Li et al. ${ }^{10}$ It has to be kept in mind that these patients were suffering from severe epilepsy and were highly selected. This group was therefore not representative for epilepsy as a whole. Nevertheless, our study underlined the value of scanning patients with localisation related epilepsy in the clinical setting. In our study 182 patients with localisation related epilepsy were not scanned at all, which is not in accordance with accepted guidelines ${ }^{12}$ and raises important clinical governance issues.

In patients with single epileptic seizures the yield of neuroimaging as used in our study was lower than in localisation related epilepsy. Several factors may explain the difference. Some patients with single attacks probably will develop IGE, patients with single attacks may have a more benign form of epilepsy that may be less likely to be associated with structural abnormalities, patients often had only CT imaging and subtle abnormalities may have been overlooked and some structural abnormalities such as atrophy may only develop after the onset of epilepsy. However, a substantial minority of patients with single seizures had abnormalities ( $18 \%)$, a result similar to the result published by King et al who found 38 lesions in 300 patients with single attacks. ${ }^{15}$ Hopkins suggested that people who have had a first seizure should be scanned if the clinical onset of the seizure was clearly partial or if there are focal neurological signs that are not clearly accounted for by known pre-existing neurological damage such as cerebral palsy or embolus. ${ }^{16}$ Following this protocol the vast majority of abnormalities would be detected. However, a small minority of potentially treatable abnormalities may be missed. In our study two patients with single seizures had tumours, one was under the age of 20 and had no localising 
signs. Another patient without localising signs had an arteriovenous malformation. Our result was in keeping with a study of 119 patients after single seizures. In three of 119 patients interventions were likely to have been both effective and stimulated by the CT scan alone rather than by the results of clinical examination-two with meningioma and one with arterio-venous malformation. ${ }^{17}$ In our study about $30 \%$ of all patients with a single unprovoked epileptic seizure had no scan. Omitting neuroimaging may be acceptable after the first seizure in patients with clear evidence for idiopathic generalised or Rolandic epilepsy, but neuroimaging should be performed in all remaining patients after the first unprovoked seizure. $^{15}$

Remission was not incompatible with structural abnormalities in our study. Most remission patients had non-specific white matter lesions and atrophy but there were patients with vascular lesions such as an arterio-venous malformation. Our study underlines the fact that remission does not exclude the presence of serious cerebral abnormalities. In our study no patient with detected hippocampal atrophy or low grade glioma was in remission.

No abnormalities were detected in patients with IGE or patients with non-epileptic seizures. Almost $40 \%$ and about $20 \%$ respectively had scans in these patient groups. Uncertainty about the diagnosis may explain why the scans were requested. Routine MRI does not add information in patients with IGE or Rolandic epilepsy and routine MRI is not recommended in those patients. ${ }^{15}$ New MRI techniques may lead to clinical applications of neuroimaging in IGE in the future ${ }^{18}$ but there is no clinical application at present. Early clinical classification, supported by EEG, is important to avoid unnecessary imaging investigations. Occasionally the EEG may show bilaterally synchronous discharges in patients with focal lesions, which may lead to the erroneous diagnosis of IGE. ${ }^{19}{ }^{20}$ The diagnosis should be reviewed and imaging performed in all patients where the seizure semiology or treatment response is unusual for IGE or Rolandic epilepsy.

The other main finding was the frequent use of CT and standard MRI with $5 \mathrm{~mm}$ thick slices in the clinical setting. About one third (163 patients) of all patients who had scans had only a cranial CT investigation, one third had a standard MRI with $5 \mathrm{~mm}$ thick slices, usually with CT (178 patients) and one third (154 patients) had a high resolution MRI including a $\mathrm{Tl}$ weighted sequence with $1.5 \mathrm{~mm}$ slice thickness. Since the initial application in $1984,{ }^{21}$ the superiority of MRI over $x$ ray CT in terms of sensitivity and specificity for identifying the aetiology of epilepsy has become firmly established. ${ }^{22-28}$ Epileptogenic lesions may be missed in patients who had only CT or standard MRI. Hippocampal sclerosis, small low grade gliomas or small arterio-venous malformations and malformations of cortical development are all difficult to detect on CT. ${ }^{9}$ In addition a CT examination of the head involves a radiation dose of $2.3 \mathrm{mSV}$, which equals more than 100 chest radiographs or the background radiation of about one year. ${ }^{29}$

MRI should therefore be the examination of choice in patients with epilepsy. CT may retain a role as the initial investigation if MRI is not possible because of claustrophobia, learning disability, acute disorders such as status epilepticus, contraindication for MRI, or if MRI is not available within reasonable time. CT also retains a role, supplementary to MRI, in the recognition of intracranial calcifications that may not be easily detected on MR images. ${ }^{30}$ Calcified tubers in tuberous sclerosis, which is commonly associated with epilepsy, are more conspicuous on CT images than MRI. This was again illustrated in our study. A calcified tuber was initially overlooked on MRI but later detected on CT. Calcifications also commonly occur in neurocysticercosis, worldwide a main cause of epilepsy. ${ }^{31}$ New MRI techniques such as magnetisation transfer imaging may increase the yield of MRI in neurocystercercosis ${ }^{32}$ and possible replace CT.
MRI is a versatile diagnostic tool and it is important to choose the appropriate imaging parameters to get optimal results in epilepsy. Important abnormalties such as hippocampal sclerosis, subtle focal cortical dysplasias, and band heterotopia may be difficult to detect on standard MRI and high resolution MRI (slice thickness $1.5 \mathrm{~mm}$ ) is the method of choice. ${ }^{334}$ The sensivity of MRI for hippocampal sclerosis can be further improved by the use of FLAIR. ${ }^{35-37}$ The limitations of standard MRI to detect hippocampal sclerosis were again highlighted in our study. The standard MRI scan of a patient with hippocampal sclerosis on postmortem examination was initially reported as normal. The recommendation to use MRI with thin slices (and preferably including a FLAIR sequence) was therefore supported by our data in the clinical setting. These MRI sequences are readily available on clinic MRI scanners and add only a few minutes to the examination. MRI and magnetic resonance spectroscopy are developing techniques. Other magnetic resonance techniques such as diffusion imaging, ${ }^{38-41}$ functional MRI, ${ }^{42}$ quantitative measurements of volume of structures and T2 relaxation time, ${ }^{43}{ }^{44}$ serial MRI, ${ }^{45}$ or spectroscopy and multi-modal imaging ${ }^{46}$ have all been used in epilepsy research and may gain a clinical role in selected epilepsy patients in the future.

\section{Summary}

In our clinical setting many patients with localisation related epilepsy had no scan or were only scanned with CT or standard MRI, which are less sensitive for important epileptogenic lesion than high resolution MRI. Our study provided further examples where high resolution MRI was superior to other imaging modalities. An MRI protocol for epilepsy should include at least one sequence with coronal Tl weighted thin slices (slice thickness $1 \mathrm{~mm}$ to $2 \mathrm{~mm}$ ).

A significant minority of patients with non-epileptic seizures and IGE were scanned but imaging did not add any relevant abnormalities.

Neuroimaging detected abnormalities in most patients with localisation related epilepsy and in a sizeable number of patients with single seizures and epilepsy in remission. Our study underlined the importance of optimal neuroimaging for these patients.

\section{ACKNOWLEDGEMENTS}

I would like to thank Dr Tom Nixon for his support and helpful comments and Professor David Chadwick for his helpful comments.

\section{REFERENCES}

1 Jackson GD, Berkovic SF, Tress BM, et al. Hippocampal sclerosis can be reliably detected by magnetic resonance imaging. Neurology 1990;40: 1869-75.

2 Cook MJ, Fish DR, Shorvon SD, et al. Hippocampal volumetric and morphometric studies in frontal and temporal lobe epilepsy. Brain 1992;115:1001-15.

3 Bronen RA, Cheung G, Charles JT, et al. Imaging findings in hippocampal sclerosis: correlation with pathology. Am J Neuroradiol $1991 ; 12: 933-40$.

4 Jack CR, Sharbrough FW, Cascino GD, et al. Magnetic resonance image-based hippocampal volumetry: correlation with outcome after temporal lobectomy. Ann Neurol 1992;31:138-46.

5 Tien RD, Felsberg GJ, Campi de Castro C, et al. Complex partial seizures and mesial temporal sclerosis: evaluation with fast spin-echo MR imaging. Radiology 1993;189:835-42.

6 Van Paesschen W, Revesz T, Duncan JS, et al. Quantitative neuropathology and quantitative magnetic resonance imaging of the hippocampus in temporal lobe epilepsy. Ann Neurol 1997;42:756-66.

7 Watson C, Andermann F, Gloor P, et al. Anatomic basis of amygdaloid and hippocampal volume measurement by magnetic resonance imaging Neurology 1992;42:1743-50.

8 Raymond AA, Fish DR, Sisodiya SM, et al. Abnormalities of gyration, heterotopias, tuberous sclerosis, focal cortical dysplasia, microdysgenesis, dysembryoplastic neuroepithelial tumour and dysgenesis of the archicortex in epilepsy. Clinical, EEG and neuroimaging features in 100 adult patients. Brain 1995;118:629-60.

9 Duncan JS. Imaging and epilepsy. Brain 1997;120:339-77.

10 Li LM, Fish DR, Sisodiya SM, et al. High resolution magnetic resonance imaging in adults with partial or secondary generalised epilepsy attending a tertiary referral unit. J Neurol Neurosurg Psychiatry 1995;59:384-7. 
11 Spencer SS. MRI and epilepsy surgery. Neurology 1995:45:1248-50.

12 Commission on neuroimaging of the international league against epilepsy. Recommendations for neuroimaging of patients with epilepsy. Epilepsia 1997;38:1255-6.

13 Commission on classification and terminology of the international league against epilepsy. Proposal for revised classification of epilepsies and epileptic syndromes. Epilepsia 1989;30:389-99.

14 Sander JWAS, Hart YM, Johnson AL, et al. National general practice study of epilepsy: new diagnosed epileptic seizures in a general population. Lancet 1990;336:1267-71.

15 King MA, Newton MR, Jackson GD, et al. Epileptology of the first-seizure presentation: a clinical, electroencephalographic, and magnetic resonance imaging study of 300 consecutive patients. Lancet 1998;26:352:1007-11.

16 Hopkins A, Garman A, Clarke C. The first seizure in adult life: value of clinical features, electroencephalography, and computerised tomographic scanning in prediction of seizure recurrence. Lancet 1988;i:721-7.

17 Schoenenberger RA, S M Heim SM. Indication for computed tomography of the brain in patients with first uncomplicated generalised seizure. BM 1994;309:986-9.

18 Woermann FG, Sisodiya SM, Free SL, et al. Quantitative MRI in patients with idiopathic generalized epilepsy. Evidence of widespread cerebral structural changes. Brain 1998;121:1661-7.

19 Tükel K, Jasper H. The electroencephalogram in parasagittal lesions. Electroencephalogr Clin Neurophysiol 1952;4:481-94.

20 Wieshmann UC, Niehaus L, Meierkord H. Ictal speech arrest and parasagittal lesions. Eur Neurol 1997;38:123-7.

21 Sostman HD, Spencer DD, Gore JC, et al. Preliminary observations on magnetic resonance imaging in refractory epilepsy. Magn Reson Imaging 1984;2:301-6

22 Cross JH, Jackson GD, Neville BG, et al. Early detection of abnormalities in partial epilepsy using magnetic resonance. Arch Dis Child 1993;69:104-109.

23 Froment JC, Mauguiere F, Fischer C, et al. Magnetic resonance imaging in refractory focal epilepsy with normal CT scans. J Neuroradiol 1989; 16:285-91.

24 Latack JT, Abou-Khalil BW, Siegel GJ, et al. Patients with partial seizures: evaluation by MR, CT, and PET imaging. Radiology 1986;159:159-63.

25 Lesser RP, Modic MT, Weinstein MA, et al. Magnetic resonance imaging (1.5 tesla) in patients with intractable focal seizures. Arch Neurol 1986;43:367-71

26 Kuzniecky R, de la Sayette V, Ethier R, et al. Magnetic resonance imaging in temporal lobe epilepsy: pathological correlations. Ann Neurol 1987:22:341-7.

27 McLachlan RS, Nicholson RL, Black S, et al. Nuclear magnetic resonance imaging, a new approach to the investigation of refractory temporal lobe epilepsy. Epilepsia 1985;26:555-62.

28 Triulzi F, Franceschi $M$, Fazio $F$, et al. Nonrefractory temporal lobe epilepsy: 1.5-T MR imaging. Radiology 1988;166:181-5.
29 Dixon AK, Dendy P. Spiral CT: how much does radiation does matter? Lancet 1998;352:1082-3.

30 Sitoh YY, Tien RD. Neuroimaging in epilepsy. J Magn Reson Imaging 1998;8:277-88.

31 Pal DK, Carpio A, Sander JW. Neurocysticercosis and epilepsy in developing countries. J Neurol Neurosurg Psychiatry 2001;68:137-43.

32 Kathuria MK, Gupta RK, Roy R, et al. Measurement of magnetization transfer in different stages of neurocysticercosis. J Magn Reson Imaging 1998:8:473-9.

33 Stevens JM. Imaging in epilepsy. Clinical MRI 1995;5:14-23.

34 McBride MC, Bronstein KS, Bennett $B$, et al. Failure of standard magnetic resonance imaging in patients with refractory temporal lobe epilepsy. Arch Neurol 1998;55:346-8.

35 Bergin PS, Fish DR, Shorvon SD, et al. Magnetic resonance imaging in partial epilepsy: additional abnormalities shown with the fluid attenuated inversion recovery (FLAIR) pulse sequence. J Neurol Neurosurg Psychiatry 1995:58:439-43.

36 Rydberg JN, Hammond CA, Grimm RC, et al. Initial clinical experience in MR-imaging of the brain with a fast fluid-attenuated inversion-recovery pulse sequence. Radiology 1994;193:173-80.

37 Wieshmann UC, Free SL, Everitt $A D$, et al. MR imaging in epilepsy with a fast FLAIR sequence. J Neurol Neurosurg Psychiatry 1996:61:357-1.

38 Wieshmann UC, Symms MR, Shorvon SD. Diffusion changes in status epilepticus. Lancet 1997;350:493-4.

39 Wieshmann UC, Clark CA, Symms MR, et al. Reduced anisotropy of water diffusion in structural cerebral abnormalities demonstrated with diffusion tensor imaging. Magn Reson Imaging 1999;17:1269-74.

40 Rugg-Gunn FJ, Eriksson SH, Symms MR, et al. Diffusion tensor imaging of cryptogenic and acquired partial epilepsies. Brain 2001;124:62736.

41 Rugg-Gunn FJ, Eriksson SH, Symms MR, et al. Diffusion tensor imaging in refractory epilepsy. Lancet 2002:359:1748-51.

42 Wieshmann UC, Krakow K, Symms MR, et al. Combined FMRI and DTI demonstrate widespread modified organisation in malformation of cortical development. J Neurol Neurosurg Psychiatry 2001;70:521-3.

43 Van Paesschen W, Revesz T, Duncan JS, et al. Quantitative neuropathology and quantitative magnetic resonance imaging of the hippocampus in temporal lobe epilepsy. Ann Neurol 1997;42:756-66.

44 Wieshmann UC, Denby CE, Eldridge PR, et al. Foramen ovale recordings: a presurgical investigation in epilepsy. Eur Neurol 2003;49:3-7

45 Lemieux L, Wieshmann UC, Moran NF, et al. The detection and significance of subtle changes in mixed-signal brain lesions by serial MR scan matching and spatial normalisation. Medical Image Analysis $1998 ; 2: 227-42$

46 Krakow K, Wieshmann UC, Woermann FG, et al. Multi modal MR imaging: functional, diffusion tensor and chemical shift imaging in a patient with localization-related epilepsy. Epilepsia 1999;40:1459-62. 